\title{
Reactivity Measurement in Estimation of Benzoquinone and Benzoquinone Derivatives' Allergenicity
}

Wilbes Mbiya ${ }^{\ddagger}$, Itai Chipinda

Department of Chemistry, Portland State University, Portland, OR 97207-0751

${ }^{\dagger}$ Allergy and Clinical Immunology Branch, Health Effects Laboratory Division,

National Institute for Occupational Safety and Health, Morgantown, West Virginia $26505-2888$

${ }^{\perp}$ Address Correspondence to:

Wilbes Mbiya, Ph.D

Portland State University

$171910^{\text {th }}$ Ave

Portland, OR 97207

wilbesm@gmail.com 


\section{Graphical Abstract}

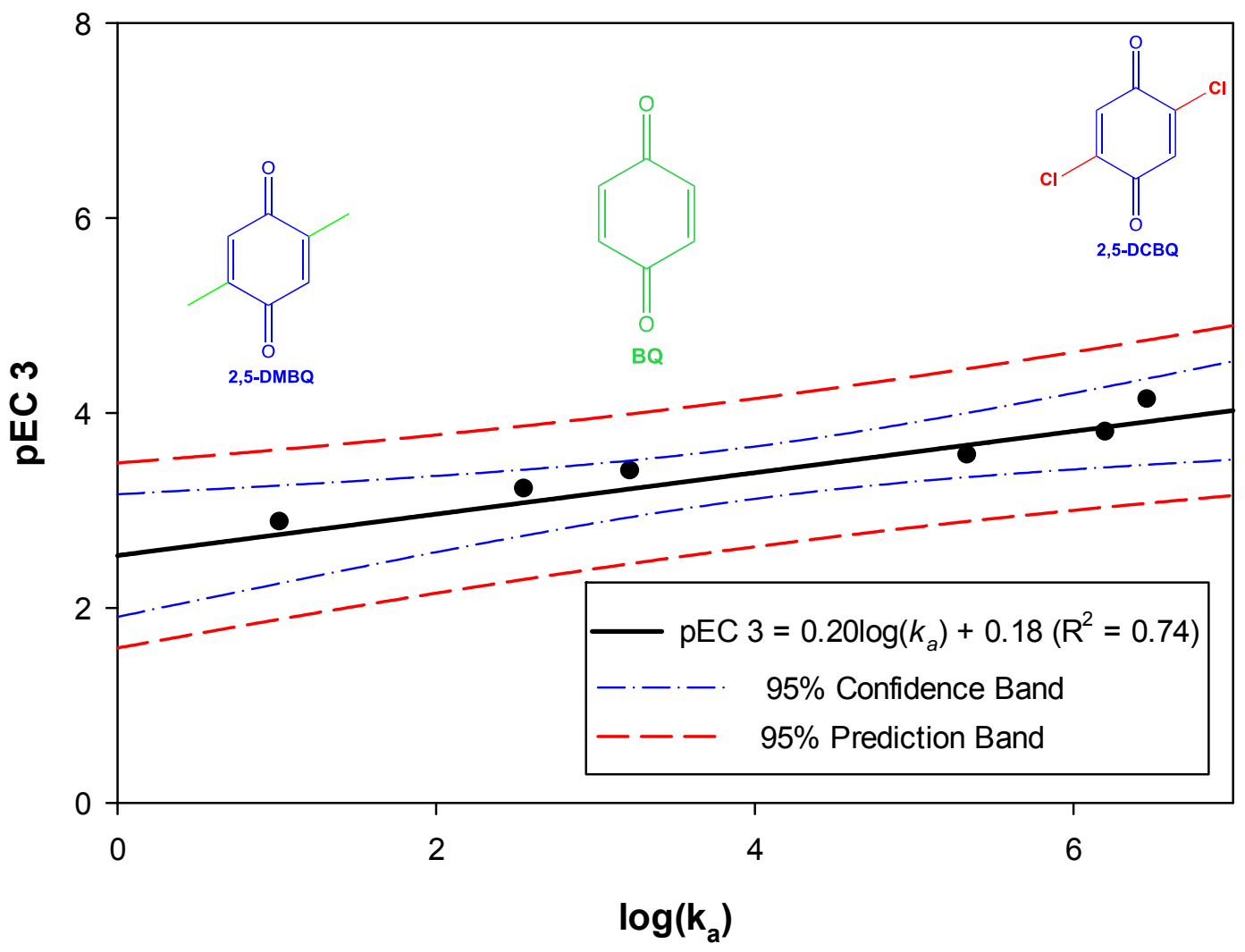




\section{ABSTRACT}

Benzoquinone (BQ) and benzoquinone derivatives (BQD) are used in the production of dyes and cosmetics. While BQ, an extreme skin sensitizer, is an electrophile known to covalently modify proteins via Michael Addition (MA) reaction whilst halogen substituted BQD undergo nucleophilic vinylic substitution (SNV) mechanism onto amine and thiol moieties on proteins, the allergenic effects of adding substituents on BQ have not been reported. The effects of inserting substituents on the BQ ring has not been studied in animal assays however mandated reduction/elimination of animals used in cosmetics testing in Europe has led to an increased need for alternatives for the prediction of skin sensitization potential. Electron withdrawing and electron donating substituents on BQ were assessed for effects on BQ reactivity toward nitrobenzene thiol (NBT). The NBT binding studies demonstrated that addition of EWG to BQ as exemplified by the chlorine substituted BQDs increased reactivity while addition of EDG as in the methyl substituted BQDs reduced reactivity. BQD with electron withdrawing groups had the highest chemical potency followed by unsubstituted BQ and the least potent were the BQD with electron donating groups. BQ and BQD skin allergenicity, was evaluated in the murine local lymph node assay (LLNA). The BQD results demonstrate the impact of inductive effects on both BQ reactivity and allergenicity, and suggest the potential utility of chemical reactivity data for electrophilic allergen identification and potency ranking.

\section{ABBREVIATIONS}

ACD, Allergic contact dermatitis; BQ, p- benzoquinone; BQD, benzoquinone derivatives; CBQ, chlorobenzoquinone; 2,5-DCBQ, 2,5-dichlorobenzoquinone, 2,6-DCBQ, 2,6-

dichlorobenzoquinone; EDG, electron donating groups; EWG, electron withdrawing groups; 
ICD, irritant contact dermatitis; LLNA, local lymph node assay; EC3, estimated concentration that produces a 3 fold increase in lymph node cell proliferation over the vehicle control; 2-MBQ, 2 methyl- benzoquinone; MA, Michael addition; NBT, nitrobenzenethiol; SI, stimulation index,; 2-tBBQ, 2-tertbutyl benzoquinone; SNV, nucleophilic vinylic substitution;

\section{INTRODUCTION}

Allergic contact dermatitis (ACD) is a prevalent occupational disease caused by a wide range of chemicals (Payne and Walsh 1994; Roberts et al. 2011). ACD which is estimated to affect 1-4\% (Smith and Hotchkiss 2001) of the general world-wide population, accounts for 30 to $>50 \%$ of occupational skin disorders depending on the industry. Over 13 million workers in the US are believed to be at risk from exposure to potential skin sensitizers and the level of compensation due to occupational contact dermatitis has been estimated to be greater than $\$ 1$ billion/yr (Mathias and Morrison 1988). ACD is also a significant health hazard of concern to developers of cosmetic, personal care, chemical, pharmaceutical, and medical device products (Mckim et al. 2010; Roberts and Aptula 2008).

Existing animal based assays such as the murine local lymph node assay (LLNA), (Basketter et al. 2000) which is based on the proliferation of lymph node cells in the induction phase or the mouse ear swelling test (MEST)(Gad 1994) and the Guinea Pig Maximization Test (GPMT)(2002) which are based on the observation of the allergic responses in the elicitation phase of ACD are widely used to identify skin sensitizing chemicals and to measure the relative sensitization potential of contact allergens. Predictive animal tests such as the LLNA that have the capacity to identify sensitizers before they are placed on the market have been highly successful (Basketter and Maxwell 2007). However, the mandated reduction/elimination of 
animals testing for cosmetics use in Europe has led to an increased need for alternatives methods for prediction of skin sensitization potential (Roberts et al. 2007). Most researchers moved to LLNA due to its animal welfare benefits while also moving to alternative non-animal test methods. For example, a published dataset consisting of quantitative test results for compounds tested in the LLNA is being used to develop and evaluate (validating or invalidating) alternative approaches (Roberts et al. 2011).

Hundreds of chemicals, belonging to different reaction mechanistic domains, have been shown to possess the ability to induce skin sensitization. One group of these chemicals is benzoquinone $(\mathrm{BQ})$ and the benzoquinone derivatives (BQD). While $\mathrm{BQ}$ and $\mathrm{BQD}$ are used in the production of dyes (Boga et al. 2013; Shimada et al. 2015) and can be reduced to hydroquinones which are used in cosmetics (Dlova et al. 2015; Matsubayashi et al. 2002; Shin and Park 2014; Uddin et al. 2011), which may result in potential skin exposure. BQ is an extreme skin sensitizer (EC3 = $0.013 \%$ (EC3 is the estimated concentration that produces a 3 fold increase in lymph node cell proliferation over the vehicle control)(Roberts and Aptula 2009) but the effects of inserting substituents on the $\mathrm{BQ}$ ring have not been studied in animal assays. BQ and BQD are known to covalently modify proteins via the Michael addition (MA) reaction and/or through nucleophilic vinylic substitution (SNV), depending on whether it is attached to either electron donating groups (EDGs) or electron withdrawing groups (EWGs) that are good leaving groups (Mbiya et al. 2012).

The seven BQD test chemicals studied with expected sites of nucleophilic attack on the ring are shown in Figure 1. The reported (Mbiya et al. 2012) reactivity constants ( $\left.k_{a}\right)$ for BQ and BQD were used to predict the LLNA EC3 values for the BQD after which LLNA studies were then performed for $\mathrm{BQ}$ and $\mathrm{BQD}$ to determine the $\mathrm{EC} 3$ values. LLNA studies were then performed 
for $\mathrm{BQ}$ and $\mathrm{BQD}$ to determine the actual $\mathrm{EC} 3$ values and test the predictive power of the reactivity data as a way of evaluating the potential utility of the reactivity approach as an alternative method for skin sensitization testing.

\section{EXPERIMENTAL SECTION}

Chemicals. $100 \mathrm{mM}$ phosphate buffer ( $\mathrm{pH}$ 7.4) was made using monosodium phosphate and disodium phosphate, acetic acid, sodium acetate, acetonitrile (ACN), 4-nitrobenzenethiol (NBT). All test chemicals were purchased from Sigma Chemical Co. (St. Louis, MO) unless otherwise stated.

\section{METHODS}

\section{Approach for QSAR Analysis}

The $k a$ values reported in Mbiya et. al 2013 were BQ and BQD were reacted with NBT were adopted. The structures of test compounds are given in Figure 1 and the arrows indicate the reaction sites. The $\log P$ values were calculated from structure using ADMET ${ }^{\circledR}$ (MedChemDesigner) Version: 2.0 software (Dearden 2007).

\section{Local lymph node assay}

Female BALB/c mice were purchased from Taconic (Hudson, NY, USA). Animals were 6-8 weeks old upon arrival and allowed to acclimate for a minimum of 10 days. Animals were housed in the Association for Assessment and Accreditation of Laboratory Animal Care International-accredited animal facility at National Institute for Occupational Safety and Health (NIOSH), Morgantown, WV, USA. Animals were housed under controlled environmental conditions in High Efficiency Particulate Act (HEPA)-filtered ventilated polycarbonate cages on 
autoclaved hardwood beta-chip bedding and provided Teklad 7913 food and autoclaved tap water ad libitum. All animal procedures were reviewed and approved by the NIOSH Animal Care and Use Committee.

The LLNA was performed according to the Interagency Coordinating Committee on the Validation of Alternative Methods (ICCVAM) standard method (Haneke et al. 2001) to determine the allergenic potency of test chemicals. The LLNA test was conducted in three independent blocks, each of which contained solvent controls, assay of 2 BQD and BQ as the positive control. After randomly grouping mice into groups $(n=4)$, mice were dosed with 25 $\mu \mathrm{L} /$ ear of a test chemical in acetone olive oil (AOO; 4:1). The negative vehicle control group was dosed with AOO. The vehicle and test chemicals were applied on the dorsum of both ears. Two hundred $\mu \mathrm{L}$ of $20 \mu \mathrm{Ci}{ }^{3} \mathrm{H}$-thymidine in $0.01 \mathrm{M}$ phosphate buffered saline was injected into the tail vein on day 6 , and after $5 \mathrm{~h}$ the mice were euthanized via $\mathrm{CO}_{2}$ inhalation. Left and right draining auricular lymph nodes were excised and pooled for each animal. Single cell suspensions were made and following overnight protein precipitation using $5 \%$ trichloroacetic acid (TCA), samples were counted on a Packard Tri-Carb 2500TR (Meriden, CT) liquid scintillation analyzer with background subtraction. Stimulation indices (SI) were calculated by dividing the mean disintegrations per minute (DPM) per test group by the mean DPM for the vehicle control group. The EC3 (effective concentration inducing a 3-fold increase in ${ }^{3} \mathrm{H}$-thymidine incorporation in the harvested lymph node cells of treated animals compared to vehicle-treated animals) was estimated by the benchmark approach, by fitting a nonlinear regression model to the data of all individual animals.

\section{Statistical Analysis}


LLNA: The structure of these experiments was a nested design. Mice were randomly placed into treatment groups, which included solvent control, positive control, + hapten groups. Lymph node cell proliferation was measured as described above. A sample size of four per treatment group provides greater than $95 \%$ power to find a change in lymphocyte proliferative activity of at least $50 \%$, based on a treatment variance of $20 \%$, which was based on previous studies. Analysis was performed using the Mixed Procedure on an SAS platform to perform a hierarchical analysis of variance. Results will be considered significant if $\mathrm{p}<0.05$. The values are given as mean $\pm \mathrm{SD}$. SigmaPlot (Version 12.0 Systat Software Inc.) was used for statistical analysis and graphical presentation (Gurevitch 1991).

\section{RESULTS}

\section{NBT-BQ/BQD Reactions}

Reaction rate constants of $\mathrm{BQ}$ and $\mathrm{BQD}$ toward NBT that were previously determined, (Mbiya et al. 2012) along with predicted EC3 values derived from equations correlating electrophilic allergen reactivity (to NBT) with pEC3, are shown in Table 1. Reactivity of BQ and BQD towards NBT was used as a surrogate for protein binding of the electrophiles in phosphate buffer at $\mathrm{pH}$ 7.4. From the rate data determined empirically, the order of reactivity toward NBT is $2,5-$ $\mathrm{DCBQ}>2,6-\mathrm{DCBQ}>2-\mathrm{CBQ}>\mathrm{BQ}>2-\mathrm{MBQ}>2-\mathrm{tBBQ}>2,5-\mathrm{DMBQ}$. There was no observable relationship between solubility $(\log \mathrm{P})$ and EC3 predicted by chemical reactivity across the entire set of test chemicals.

Prediction of EC3 values from Reactivity Data. The $k_{a}$ values taken from Table 1 were used to predict EC3 values using the linear equation A1 which was derived by Chipinda et. al. (Chipinda 
et al. 2010) using a structurally diverse MA domain data set where $\mathrm{pEC} 3$ is obtained by dividing the molecular weight $(M w t)$ of the chemical by EC3 value and calculating the log (A2). The results were compared with EC3 values obtained from the LLNA performed for these BQD.

$$
\begin{gathered}
p E C 3=0.81 \log k_{a}+2.13 \\
p E C 3=\log \left(\frac{M w t}{E C 3}\right)
\end{gathered}
$$

The presence of the EDG on the BQ reduces the predicted LLNA potency of BQ (predicted EC3; $0.002 \%)$ from an extreme sensitizer to a strong sensitizer (2,5-DMBQ; predicted EC3 $=1.28 \%$ ). Addition of an EWG increased the reactivity and thus the relative predicted potency rating of BQ (Table 1).

\section{LLNA: Skin sensitization assessment}

The LLNA was performed for BQ to derive a more accurate EC3 estimate rather than use the literature EC3 value for BQ which was extrapolated from doses that produced high stimulation indexes (SI) well above the three-fold threshold (Chipinda et al. 2010). BQ was then used as the positive control for all subsequent LLNA studies. Figures $2 \mathrm{a}$ and $2 \mathrm{~b}$ show the doses of each BQD and the stimulation indexes. The LLNA EC3 values estimation was conducted following $\log$ transformation of the SI data. BQ and chlorine substituted BQ allergenic potency increased in the following order: $\mathrm{BQ}(\mathrm{EC} 3=0.043 \%)<\mathrm{CBQ}(\mathrm{EC} 3=0.039 \%)<2,6-\mathrm{DCBQ}(\mathrm{EC} 3=0.028$ $\%)<2,5$-DCBQ $(\mathrm{EC} 3=0.013 \%)$ in agreement with the trend predicted by rate constants (listed in Table 1). Methyl substituted BQ EC3 values which ranged from $<0.1 \%$ to $0.238 \%$ were higher than those of chloro BQD. For 2-MBQ and 2,5-DMBQ the LLNA EC3s predicted by reactivity were different from the experimentally determined the EC3s. On the basis of reactivity 
alone all the $\mathrm{BQD}$, except 2,5-DMBQ, were predicted to be extreme sensitizers. While reactivity data predicted 2,5-DMBQ to have an 18-fold higher EC3 than 2-MBQ, the experimental EC3 values of these two electrophilic chemicals were similar to each other, but in the reverse order than predicted. However, the $10 \% 2-\mathrm{MBQ}$ dose $(\mathrm{SI}=31.6 \%)$ produced a statistically significant higher SI $(\mathrm{P}<0.001)$ than the $10 \%$ 2,5-DMBQ dose $(\mathrm{SI}=11.4 \%)$ suggesting that it may actually be a more potent allergen than 2,5-DMBQ as predicted from the reactivity data (Figure 2b). 2tBBQ EC3 could not be estimated using the LLNA due to systemic toxicity observed and lack of dose-dependency of the lymph node cell proliferation in dosed mice. In general, the predictive equation of Chipinda et al. (Chipinda et al. 2010) was generated from data derived from multiple structurally diverse chemicals with reaction rates which were lower than those of BQ. This resulted in consistent overestimation of the potency of the BQD series.

Correlation analysis of reactivity data with LLNA EC3 values. The pEC3 determined from LLNA SI values for the test chemicals were plotted against $\log k_{a}$ values to determine the relationship of reactivity to chemical skin sensitization potency. A plot of pEC3 values for 6 test chemicals is shown in Figure 3. A strong positive correlation $\left(\mathrm{R}^{2}=0.74\right)$ between potency in LLNA and reactivity was observed as indicated by the following linear equation A3 (with statistical parameters);

$$
\begin{aligned}
& \text { pEC } 3=0.20( \pm 0.05) \log \left(k_{a}\right)+0.18( \pm 0.33) \\
& \mathrm{n}=6, \mathrm{R}^{2}=0.74, \mathrm{R}^{2} \text { adj }=0.68, \mathrm{~s}=0.27 \text { and } \mathrm{F}=11.6
\end{aligned}
$$

It must be stated that due to the limited number of the test chemicals used in this study conclusions concerning the potential relationship between mechanism of chemical reactivity and 
allergenic potency cannot be made from these results. $\mathrm{R}^{2}$ of 0.74 between the overlapping data of MA and SNV domains suggests that reactivity rate has greater influence than chemical mechanistic domain toward a chemical's allergenic potency (Mbiya et al. 2012).

\section{DISCUSSION}

The BQ moiety common to all test chemicals within this series was considered important to allow an accurate comparison of the reactivity to NBT as well as allergenic potency in the LLNA, while avoiding potential confounders that may arise from structural differences. From the LLNA data and prediction of EC3 values using rate constants, we observed that BQD with EWGs had the highest chemical potency, followed by unsubstituted BQ, and the least potent were the BQD with EDGs. This trend agreed well with what was expected because electrondonating substituents, such as methyl groups, reduce reactivity while electron withdrawing substituents, such as halogens, increase reactivity of compounds in the MA domain (Aptula and Roberts 2006). It is particularly striking that relatively small changes in the BQ structure have a dramatic impact on the LLNA EC3. This is observed when the LLNA EC3 decreases with increasing number of chlorine atoms added to $\mathrm{BQ}(\mathrm{BQ}>\mathrm{CBQ}>\mathrm{DCBQ})$. The effect of different positions on reactivity is observed between 2,5-DCBQ and 2,6-DCBQ in which 2,5-DCBQ (EC3 $=0.013 \%)$ was 2.15 times more reactive than 2,6-DCBQ $(\mathrm{EC} 3=0.028 \%)$. This is because the para-substituted EWG inductive influence on the leaving group is greater than the meta substituted EWG (Mbiya et al. 2012).

The CBQ was less reactive than the DCBQ because both EWGs on DCBQ contribute to the inductive effect versus the effect of one EWG on the CBQ. The other reason why DCBQs are 
apparently more reactive than CBQ is because the DCBQs bind at a 1:2 ratio with protein thiols versus the 1:1 ratio protein thiol binding of CBQ (Mbiya et al. 2012).

The $10 \%$ dose 2-MBQ is more potent than 2,5-DMBQ that is more consistent with reactivity, not solubility being the driving force. The LLNA results demonstrated that hydrophobicity might be an important factor in the potency unlike in the reactivity rate model where absorption rates are not factored in. As observed in LLNA results, ranking from extreme to strong sensitizers seems to suggest that the rate of penetration role is increasing as the BQD become more hydrophobic (Table 1). This ranking from extreme to strong noticed in the experiments was absent from the predicted EC3 values demonstrating that hydrophobicity plays a very minor role if we are to determine EC3 values based on reactivity alone. The predictions done using equation A1 overestimate the potency by assuming that the penetration rates $\left(k_{p}\right)$ (hydrophobicity) are similar across the whole BQD series. From the LLNA data, hydrophobicity evidently plays a role even though reactivity was still the dominant factor. This is demonstrated by the fact that the EC3 values for the $\mathrm{Cl}$ substituted BQD classify them as extreme sensitizers and these have higher $\log \mathrm{P}$ values compared to Me- substituted BQD. The large discrepancy observed between the predicted and actual EC3 values may also be due to the fact that the potency in MA domain is less strongly dependent on reactivity alone than other domains. The systemic toxicity we observed with tBBQ may be a result of increased $k_{p}$ such that more of the tBBQ is passing through the skin than can be haptenated to proteins.

We previously reported the trend in the electron magnetic resonance signal of these test chemicals to be $\mathrm{BQ}<\mathrm{CBQ}<2,6-\mathrm{DCBQ}<2,5-\mathrm{DCBQ}$ (Mbiya et al. 2012). This is the same trend observed in the LLNA EC3 values of the present study. Taken together with the present data we concluded that BQ radical might play a minor role in haptenation of proteins. This 
agrees well with what was previously suggested by Christensson et al. (2006), in that semiquinone radicals may act as a hapten.

\section{CONCLUSIONS}

The high correlation between chemical reactivity as demonstrated empirically using the NBT assay and the LLNA threshold EC3 estimates (Table 1) of the BQ and BQD series suggests there is potential utility of chemical reactivity for electrophilic allergen identification and potency ranking and that the rate of chemical-to-protein binding is a major determinant of skin allergic sensitization potency. Influence of other physical-chemical factors such as log P, mechanistic domain, and haptenated protein stability on a chemical's skin sensitization potency remains to be determined and may vary between chemical mechanistic classes.

\section{Acknowledgments}

The findings and conclusions in this report are those of the authors and do not necessarily represent the views of the National Institute for Occupational Safety and Health.

\section{Funding}

This work was supported by an Interagency Agreement with the NIEHS (IAG\#Y1-ES-0001-12) and Grant Number CHE 1056311 from the National Science Foundation. 


\section{Reference List}

1. Standard Practice for Testing Guinea Pigs for Contact Allergens: Guinea Pig Maximization Test. F 720 - 81. 2002. West Conshohocken, American Society for Testing and Materials.

2. Aptula, A. O., and Roberts, D. W. (2006). Mechanistic applicability domains for nonanimalbased prediction of toxicological end points: General principles and application to reactive toxicity. Chem. Res. Toxicol. 19(8), 1097-1105.

3. Basketter, D., and Maxwell, G. (2007). In vitro approaches to the identification and characterization of skin sensitizers. Cutaneous and Ocular Toxicology 26(4), 359-373.

4. Basketter, D. A., Balikie, L., Dearman, R. J., Kimber, I., Ryan, C. A., Gerberick, G. F., Harvey, P., Evans, P., White, I. R., and Rycroft, R. J. (2000). Use of the local lymph node assay for the estimation of relative contact allergenic potency. Contact Dermatitis 42(6), 344-348.

5. Boga, C., Delpivo, C., Ballarin, B., Morigi, M., Galli, S., Micheletti, G., and Tozzi, S. (2013). Investigation on the dyeing power of some organic natural compounds for a green approach to hair dyeing. Dyes and Pigments 97(1), 9-18.

6. Chipinda, I., Ajibola, R. O., Morakinyo, M. K., Ruwona, T. B., Simoyi, R. H., and Siegel, P. D. (2010). Rapid and Simple Kinetics Screening Assay for Electrophilic Dermal Sensitizers Using Nitrobenzenethiol. Chem. Res. Toxicol. 23(5), 918-925.

7. Christensson, J. B., Matura, M., Backtorp, C., Borje, A., Nilsson, J. L. G., and Karlberg, A. T. (2006). Hydroperoxides form specific antigens in contact allergy. Contact Dermatitis 55(4), 230237.

8. Dearden, J. C. (2007). In silico prediction of ADMET properties How far have we come? Expert Opinion on Drug Metabolism \& Toxicology 3(5), 635-639.

9. Dlova, N. C., Hamed, S. H., Tsoka-Gwegweni, J., and Grobler, A. (2015). Skin lightening practices: an epidemiological study of South African women of African and Indian ancestries. British Journal of Dermatology 173, 2-9.

10. Gad, S. C. (1994). The mouse ear swelling test (MEST) in the 1990s. Toxicology 93(1), 33-46.

11. Gurevitch, J. (1991). Sigma-Plot - Scientific Graphing Software. Quarterly Review of Biology 66(1), 115-116.

12. Haneke, K. E., Tice, R. R., Carson, B. L., Margolin, B. H., and Stokes, W. S. (2001). ICCVAM evaluation of the murine local lymph node assay. Data analyses completed by the National Toxicology Program Interagency Center for the Evaluation of Alternative Toxicological Methods. Regul. Toxicol Pharmacol. 34(3), 274-286.

13. Mathias, C. G., and Morrison, J. H. (1988). Occupational skin diseases, United States. Results from the Bureau of Labor Statistics Annual Survey of Occupational Injuries and Illnesses, 1973 through 1984. Arch. Dermatol. 124(10), 1519-1524. 
14. Matsubayashi, T., Sakaeda, T., Kita, S., Nara, M., Funasaka, Y., Ichihashi, M., Fujita, T., Kamiyama, F., Yamamoto, A., Nordlund, J. J., Kaneko, M., Iida, A., Hirai, M., and Okumura, K. (2002). Pharmaceutical and clinical assessment of hydroquinone ointment prepared by extemporaneous nonsterile compounding. Biological \& Pharmaceutical Bulletin 25(1), 92-96.

15. Mbiya, W., Chipinda, I., Siegel, P. D., Mhike, M., and Simoyi, R. H. (2012). Substituent Effects on the Reactivity of Benzoquinone Derivatives with Thiols. Chem. Res. Toxicol. 26(1), 112-123.

16. Mckim, J. M., Keller, D. J., and Gorski, J. R. (2010). A new in vitro method for identifying chemical sensitizers combining peptide binding with ARE/EpRE-mediated gene expression in human skin cells. Cutaneous and Ocular Toxicology 29(3), 171-192.

17. Payne, M. P., and Walsh, P. T. (1994). Structure-Activity-Relationships for Skin Sensitization Potential - Development of Structural Alerts for Use in Knowledge-Based Toxicity Prediction Systems. Journal of Chemical Information and Computer Sciences 34(1), 154-161.

18. Roberts, D. W., and Aptula, A. O. (2008). Determinants of skin sensitisation potential. Journal of Applied Toxicology 28(3), 377-387.

19. Roberts, D. W., and Aptula, A. O. (2009). Does the extreme skin sensitization potency of benzoquinone result from special chemistry? Contact Dermatitis 61(6), 332-336.

20. Roberts, D. W., Aptula, A. O., and Patlewicz, G. (2007). Electrophilic chemistry related to skin sensitization. Reaction mechanistic applicability domain classification for a published data set of 106 chemicals tested in the mouse local lymph node assay. Chem. Res. Toxicol. 20(1), 44-60.

21. Roberts, D. W., Aptula, A. O., and Patlewicz, G. Y. (2011). Chemistry-Based Risk Assessment for Skin Sensitization: Quantitative Mechanistic Modeling for the SNAr Domain. Chem. Res. Toxicol. 24(7), 1003-1011.

22. Shimada, Y., Horiguchi-Babamoto, E., and Matsumoto, S. (2015). Unexpected photochemical debenzylation of 2,5-bis(dibenzylamino)-3,6-dichloro-p-benzoquinone. Dyes and Pigments 121, 336-341.

23. Shin, J. W., and Park, K. C. (2014). Current clinical use of depigmenting agents. Dermatologica Sinica 32(4), 205-210.

24. Smith, C. K., and Hotchkiss, S. A. M. (2001). Allergic Contact Dermatitis; Chemical and Metabolic Mechanisms, Taylor and Francis, London.

25. Uddin, S., Rauf, A., Kazi, T. G., Afridi, H. I., and Lutfullah, G. (2011). Highly sensitive spectrometric method for determination of hydroquinone in skin lightening creams: application in cosmetics. International Journal of Cosmetic Science 33(2), 132-137. 


\section{Figure Legions}

Figure 1: BQ and BQD test chemicals and their corresponding observed EC3 values. The arrows are the sites of nucleophilic attack.

Figure 2 Murine local lymph node assay (LLNA) for BQ and BQD (a): BQ(0.043\%), 2ClBQ(0.039\%), 2,5-DClBQ(0.013\%) and 2,6-DCBQ(0.028\%), (b): 2-MBQ(0.238\%), 2,6DMBQ $(0.181 \%)$ and $\mathrm{tBBQ}(--\%)$. tBBQ produced clinical signs of systemic toxicity including weight loss, lethargy and ruffled fur that gave poor dose-dependent proliferative responses and preventing a reliable EC3 determination. $\left(^{*}\right)$ the 10\% dose SI for 2-MBQ is greater than 2,5DMBQ $(\mathrm{P}<0.001)$.

Figure 3: Correlation of pEC 3 estimated using the LLNA vs. $\log$ ka of BQ and BQD from both MA and SNV reactivity domains. pEC3 was LLNA estimates and log ka was derived from reactivity toward NBT as previously reported (Roberts et al. 2007). 
Table 1: Predicted EC3 Values from Reactivity Data

\begin{tabular}{|l|l|l|c|c|c|c|}
\hline Chemical & $\begin{array}{l}\text { Mwt } \\
(\mathbf{g} / \mathbf{m o l})\end{array}$ & $\boldsymbol{k}_{a}\left(\mathbf{s}^{-1}\right)$ & $\mathbf{p E C 3}$ & $\begin{array}{c}\text { Predicted EC3 } \\
(\boldsymbol{\%})^{* 1}\end{array}$ & $\begin{array}{c}\text { LLNA } \\
\text { derived } \\
\mathbf{E C 3}(\boldsymbol{\%})\end{array}$ & Log P \\
\hline 2,5-DCBQ & 176.9 & $2.92 \times 10^{6}$ & 7.37 & $7.61 \times 10^{-6}$ & 0.013 & 1.341 \\
\hline 2-CBQ & 142.5 & $2.17 \times 10^{5}$ & 6.45 & $4.82 \times 10^{-5}$ & 0.039 & 0.980 \\
\hline 2,6-DCBQ & 176.9 & $1.60 \times 10^{6}$ & 7.16 & $1.24 \times 10^{-5}$ & 0.028 & 1.338 \\
\hline BQ & 108.1 & $1.66 \times 10^{3}$ & 4.74 & $1.97 \times 10^{-3}$ & 0.043 & 0.497 \\
\hline 2-MBQ & 122.1 & $1.51 \times 10^{3}$ & 4.23 & $7.24 \times 10^{-2}$ & 0.238 & 0.752 \\
\hline 2-tBBQ & 164.2 & $3.60 \times 10^{2}$ & 3.25 & $9.31 \times 10^{-2}$ & ---- & 1.998 \\
\hline 2,5-DMBQ & 136.2 & $1.05 \times 10^{1}$ & 2.03 & 1.28 & 0.181 & 1.070 \\
\hline
\end{tabular}

${ }^{*}$ Estimations of EC3 were performed using the regression equation $\mathrm{pEC} 3=0.81 \log \mathrm{ka}+2.13$ as reported by Chipinda et. al. ${ }^{19}$ where $p E C 3=\log ($ Mwt/EC3 $)$. 


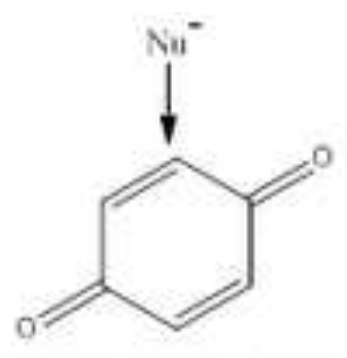

(I) p-benzoquinone $(B Q ; 0.043)$

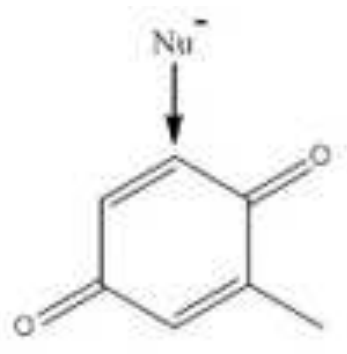

(2) 2-methy1-p-benzoquineac (2-MBQ $; 0,238)$

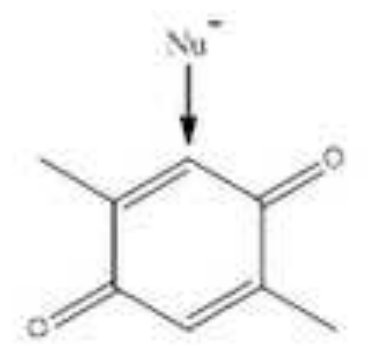

(3) 2.5-dimethyl-p-benroquinone (2.5-1)MBQ ; 0.181 )

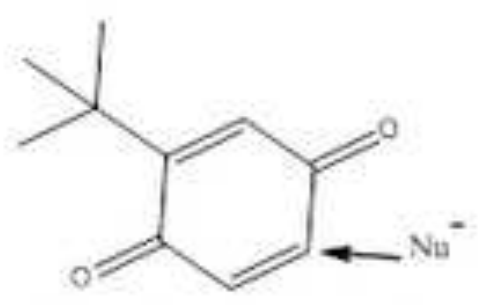

(4) 2-tert-butyl-p-benzoquinone (BBBQ)
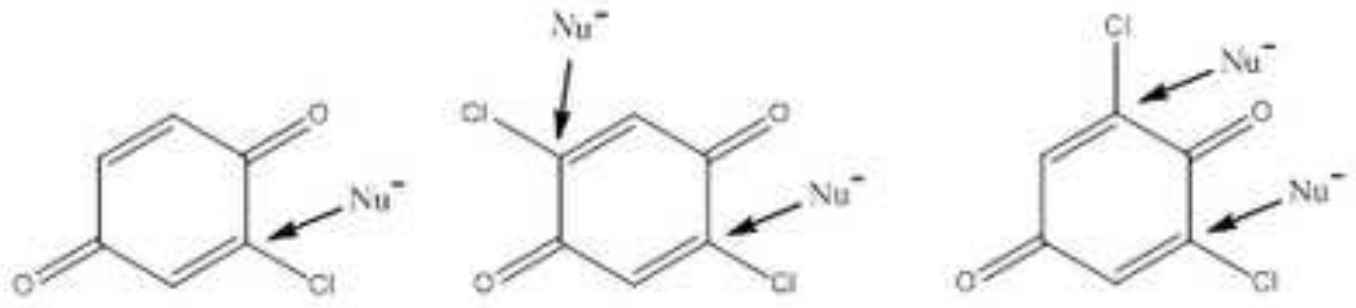

(5) 2-chloro-p-bencoquinoae (6) 2,5-4fichloro-p-benfoquinune (7) 2,6-dichlore-p-benzouguinone (2. CBQ $\div 0.039$ )

(2.5-DCPO: 0.013)

(2,6-DCBQ; 0.028) 


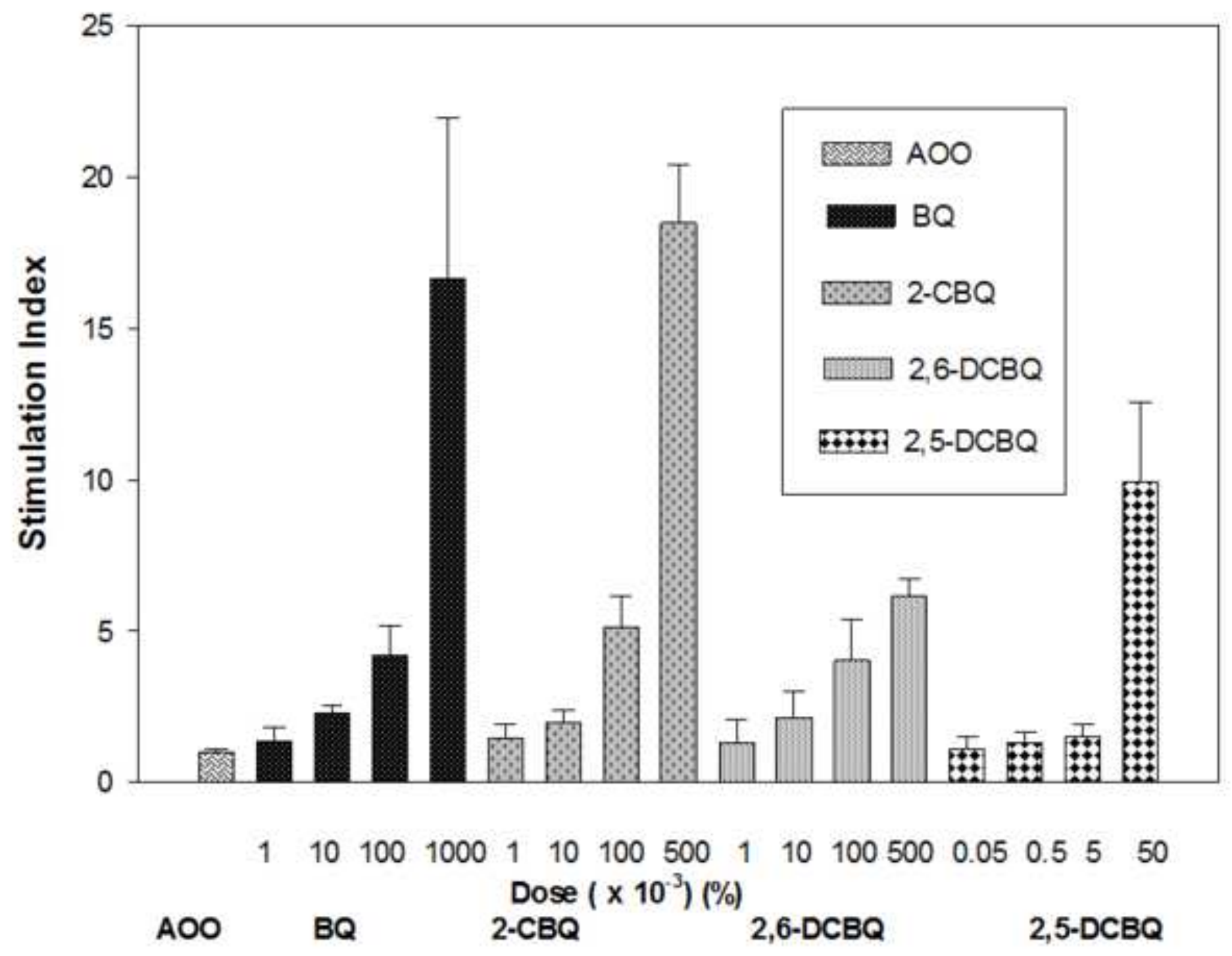




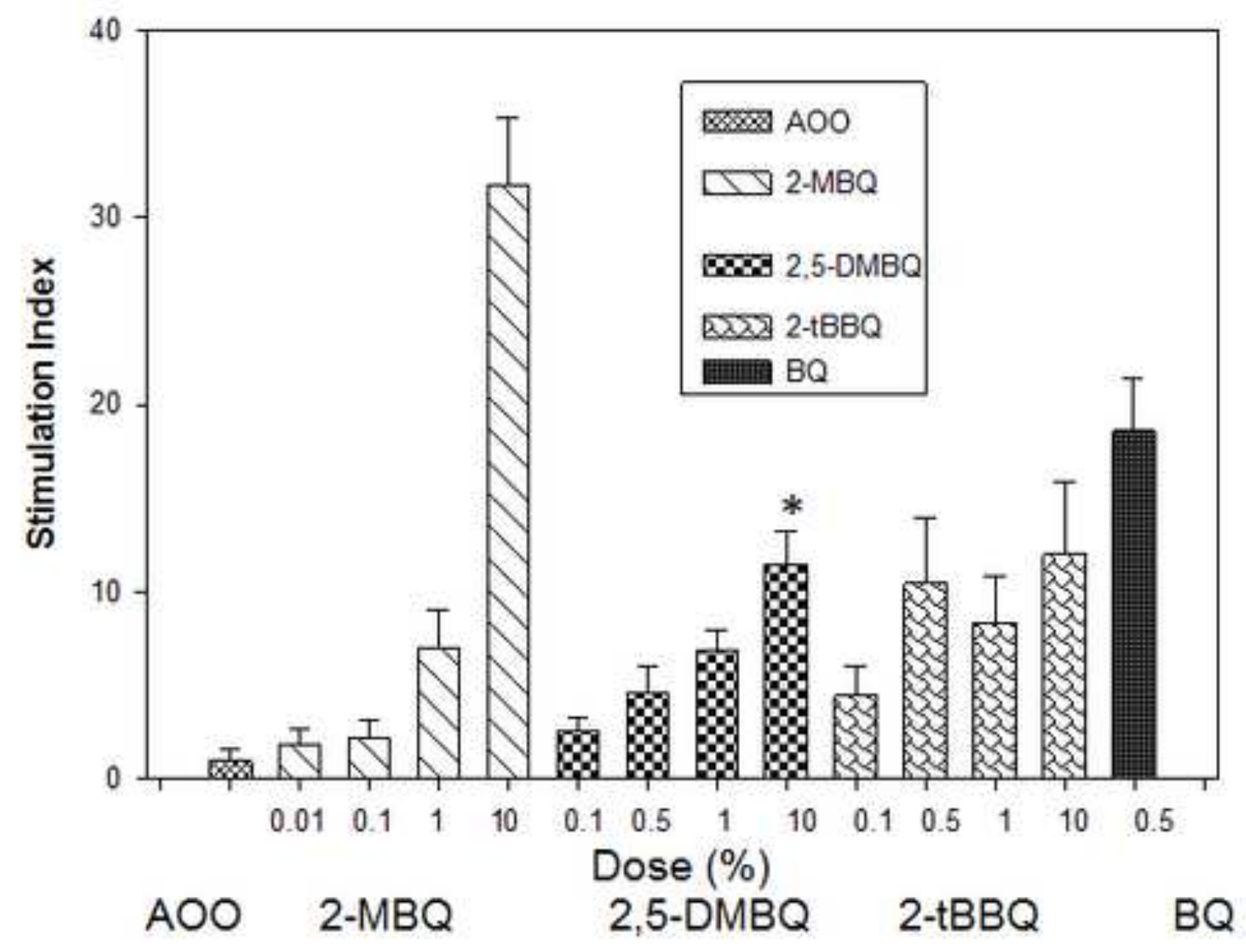




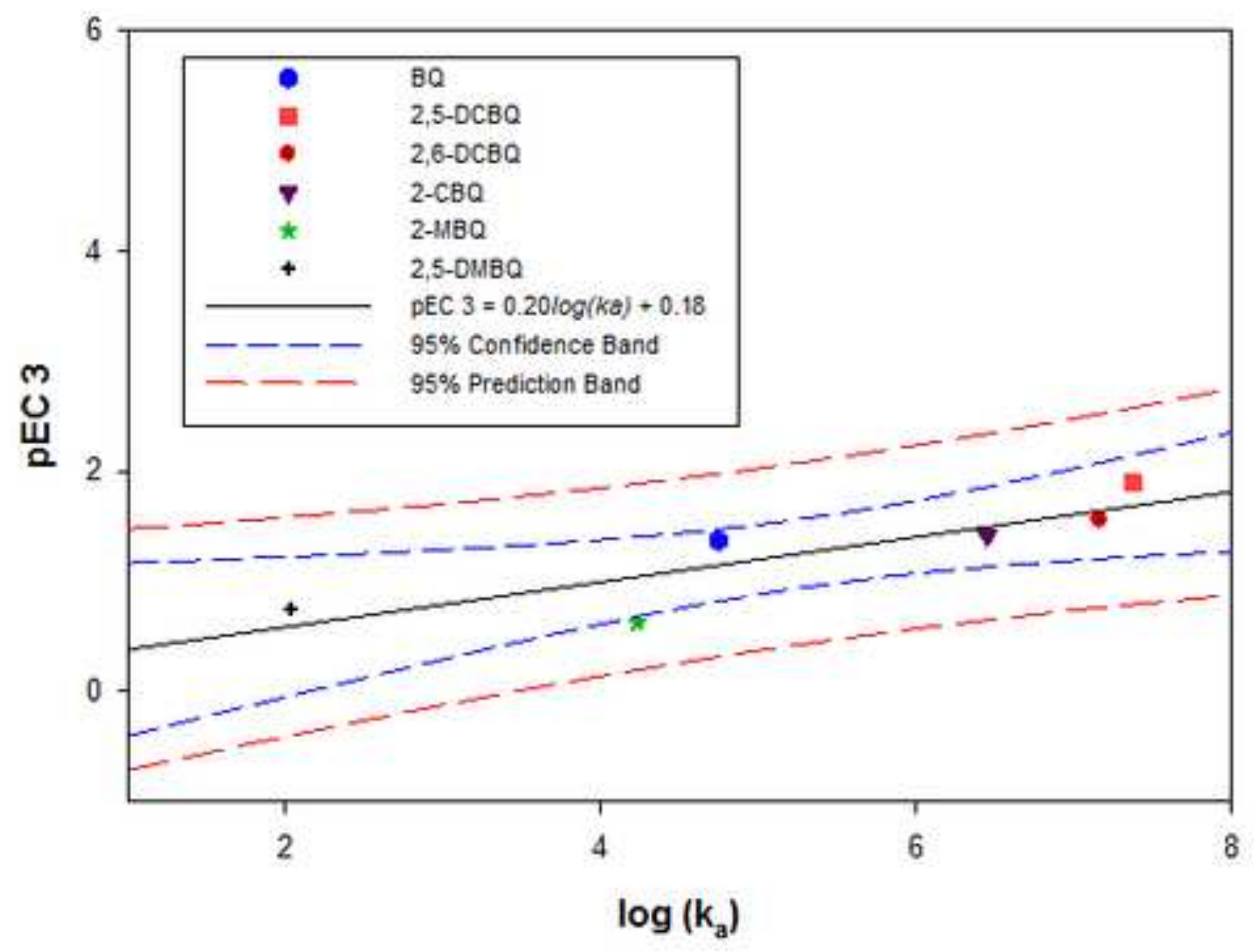

